\title{
Flow Partitioning in Rectangular Open Channel Flow
}

\author{
Yu Han $(\mathbb{D}),{ }^{1}$ Shu-Qing Yang $\left(\mathbb{D},{ }^{2}\right.$ Muttucumaru Sivakumar $\left(\mathbb{D},{ }^{2}\right.$ \\ Liu-Chao Qiu $\left(\mathbb{D},{ }^{1}\right.$ and Jian Chen $\mathbb{1}^{3}$ \\ ${ }^{1}$ College of Water Resources \& Civil Engineering, China Agricultural University, Beijing 100083, China \\ ${ }^{2}$ School of Civil, Mining \& Environmental Engineering, University of Wollongong, Wollongong, NSW 2522, Australia \\ ${ }^{3}$ College of Engineering, China Agricultural University, Beijing 100083, China
}

Correspondence should be addressed to Jian Chen; jchen@cau.edu.cn

Received 16 October 2017; Accepted 14 March 2018; Published 6 May 2018

Academic Editor: Marco Pizzarelli

Copyright (C) 2018 Yu Han et al. This is an open access article distributed under the Creative Commons Attribution License, which permits unrestricted use, distribution, and reproduction in any medium, provided the original work is properly cited.

\begin{abstract}
Hydraulic engineers often divide a flow region into subregions to simplify calculations. However, the implementation of flow divisibility remains an open issue and has not yet been implemented as a fully developed mathematical tool for modeling complex channel flows independently of experimental verification. This paper addresses whether a three-dimensional flow is physically divisible, meaning that division lines with zero Reynolds shear stress exist. An intensive laboratory investigation was conducted to carefully measure the time-averaged velocity in a rectangular open channel flow using a laser Doppler anemometry system. Two innovative methods are employed to determine the locations of division lines based on the measured velocity profile. The results clearly reveal that lines with zero total shear stress are discernible, indicating that the flow is physically divisible. Moreover, the experimental data were employed to test previously proposed methods of calculating division lines, and the results show that Yang and Lim's method is the most reasonable predictor.
\end{abstract}

\section{Introduction}

A reliable assessment of the distribution of boundary shear stresses in turbulent open channel flow is of vital importance for analyzing various critical engineering problems such as channel design, sediment transport, environmental load, and wetland design [1]. Currently, researchers directly determine boundary shear stresses by measuring the turbulence structures in the viscous sublayer, where accurate data is very difficult to acquire $[2,3]$. Thus, it is essential to develop a novel method for accurately determining the boundary shear stress using main flow data that is relatively easy to acquire.

From this perspective, the theory of flow partitioning, which is widely accepted to be an effective mathematical tool for simplifying hydraulic calculations, would be instrumental in the development of the proposed novel method. Leighly [4] was the first to propose that a flow region is divisible based on its isovels and orthogonal trajectories. Chiu and his colleagues [5-7] further developed this theory by proposing a mathematical model to describe isovel patterns. However, Nezu and Nakagawa [8] noted that the description of isovel curves by means of simple analytical functions introduces large errors. Hence, the implementation of flow divisibility remains an open issue and has not yet been implemented as a fully developed mathematical tool for modeling complex channel flows independently of experimental verification.

Since the theory of a divisible flow region was first proposed, efforts have been made to determine the location of division lines within the flow. The Keulegan method (KM; [9]) assumes that the division line is the bisector of the base angles of a polygonal channel and that it is applicable only to a channel with equal roughness on the sidewalls and bed. Einstein [10] also extended the theory of divisibility to river flows by assuming that the cross section of a river can be separated into three regions. While Chien and Wan [11] attempted to explain Einstein's hypothesis in terms of the energy transport mechanism, they did not provide a useful method of determining the division lines. Hence, this work has received little attention since its publication. In addition, a number of models have been proposed to express the division lines within a divisible flow, for example, Daido's method (DM; [12]), Guo and Julien's method (GJM; [13]), 
and Yang and Lim's method (YLM; [14]). These models were subjected to detailed reviews by Yang et al. [15] and Han et al. [16, 17], and these authors concluded that division lines could be located based on the existence of measured Reynolds shear stress values of zero below the free surface in rectangular channel flow. However, the Reynolds shear stress can only be measured using very sophisticated equipment, like laser Doppler anemometry (LDA), in controlled laboratory environments. Thus, researchers urgently require a novel method to determine the existence of division lines using a common parameter (e.g., the time-averaged velocity) that can be measured using relatively simple equipment. More importantly, practicing engineers can employ this new method to infer whether the flow region in their study is divisible.

Based on these considerations, the present study seeks to determine the existence of division lines by analyzing the mean velocity distribution in an open channel rectangular flume. In this study, two methods were developed to locate division lines: the first employs the condition of total zeroshear stress and the second employs the log-law. We experimentally verify the existence of division lines in an open rectangular channel and validate the two proposed methods.

\section{Classic Log-Law and Implications of Shear Stress}

2.1. Classic Log-Law and Empirical Determination of Its Parameters. The log-law is one of the most important and reliable laws in turbulent flow and has been demonstrated to accurately reflect the velocity of fluid flow near the boundary of the fluid region (i.e., the wall). In the wall region, the characteristic scales of length and velocity are represented using the inner variables $v / u_{*}$ and $u_{*}$, respectively, where $v$ is the kinematic viscosity and $u_{*}$ is the shear velocity. In this region, the total shear stress layer is nearly constant and equal to $\rho u_{*}{ }^{2}$, where $\rho$ is the fluid density. Based on this, Prandtl [18] expressed the momentum flux caused by secondary currents $\rho \overline{u^{\prime} v_{n}^{\prime}}$ in terms of the mixing length $l_{m}$, as follows:

$$
-\overline{\rho u^{\prime} v^{\prime}}=\rho l_{m}^{2}\left|\frac{d u}{d y}\right| \frac{d u}{d y}=\rho u_{*}^{2},
$$

where $u$ is the time-averaged velocity along the wall and $l_{m}$ is assumed to be proportional to the distance $y$ to the boundary, that is, $l_{m}=\kappa y$. Here, $\kappa=0.4$ is an empirical parameter denoted as the von Karman constant. Integration of (1) with the boundary condition that $u=0$ at the wall position $y=y_{0}$, yields the well-known logarithmic velocity profile [19]:

$$
\frac{u}{u_{* 1}}=\frac{1}{\kappa} \ln \frac{y}{y_{0}} .
$$

In the standard expression of the log-law, $u_{* 1}$ is the global shear velocity, and $y_{0}$ is empirically expressed by Nikuradse [20] as follows:

$$
y_{0}=\frac{v}{u_{* s}}
$$

where $c$ is an experimentally determined coefficient and $u_{* s}$ is the local shear velocity, which may differ from $u_{* 1}$. For circular pipe flow under the force of gravity $g$, it is well known that $u_{* 1}=u_{* s}=(g R S)^{0.5}$, where $R$ is the hydraulic radius, and $S$ is the channel slope. This empirical expression has been widely extended to channel flows. However, disagreements with experimental data have induced researchers to modify the log-law, and numerous alternatives have been proposed. For example, $\kappa$ has been treated as a variable, or $c$ has been made to vary according to flow conditions. Moreover, additional terms have been included, such as in the log-wake law. A comprehensive review of these modifications can be found in the literature (e.g., Guo [2] and Nezu and Nakagawa [8]).

In contrast to the empirical modifications discussed above, Tracy and Lester [21] first questioned the definition of shear velocities using experimental data obtained from central channel profiles. Their results showed that, for open channel flows, the value of $u_{* 1}$ employed on the left hand side of the log-law given by (2) is not identical to the value of $u_{* s}$ by which $y_{0}$ is defined in (3), but, rather, $u_{* 1}=$ $(g R S)^{0.5}$ and $u_{* s}=(g h S)^{0.5}$, where $h$ is the water depth. Surprisingly, the measured data agreed very well with the log-law without the introduction of any new terms, like the wake-law term. Furthermore, Yang et al. [22] reexamined experimentally measured data in the literature obtained for a rectangular duct flow and rectangular open channel flows with various aspect ratios. They verified that Tracy and Lester's [21] conclusion was valid even in the corner regions of channel flows when $u_{* s}=\sqrt{g L_{n} S}$, where $L_{n}$ denotes the normal distance from the boundary to the division line. This establishes a relationship between the mean velocity and the division line, and a method to test whether a flow region is divisible can be thereby developed based on the local boundary shear stress according to a single parameter $L_{n}$. However, owing to the difficulties involved in the direct measurement of the local shear stress, it would be ideal if $u_{* s}$ could be determined from the velocity distribution. Thus, it is worth investigating how to determine $u_{* s}$ from a velocity profile.

As indicated by $(2), u_{* 1} / \kappa$ can be generally evaluated from the slope of a plot of the measured mean velocity against $\ln y_{n}$ (i.e., the distance to the boundary in the normal direction) based on the measured velocity profile. In addition, $u_{* s}$ can be evaluated from its vertical axis intercept. Finally, the values of $u_{* s}$ obtained from the velocity profiles can be employed to determine the characteristic distance $L_{n}$ in an open channel flow as $L_{n}=u_{* s}^{2} /(g S)$. Therefore, the upper boundary of the subregion or division line can be inferred from the location of $L_{n}$. This forms the basis of the first simple method proposed in the present study to determine division lines in a channel flow.

2.2. Inferred Division Lines from the Zero Total Shear Stress (ZTSS) Condition. The total shear stress $\tau$ along a division line is expressed in the following form:

$$
\tau=\mu \frac{\partial u}{\partial n}-\rho u v_{n}-\rho \overline{u^{\prime} v_{n}^{\prime}}
$$




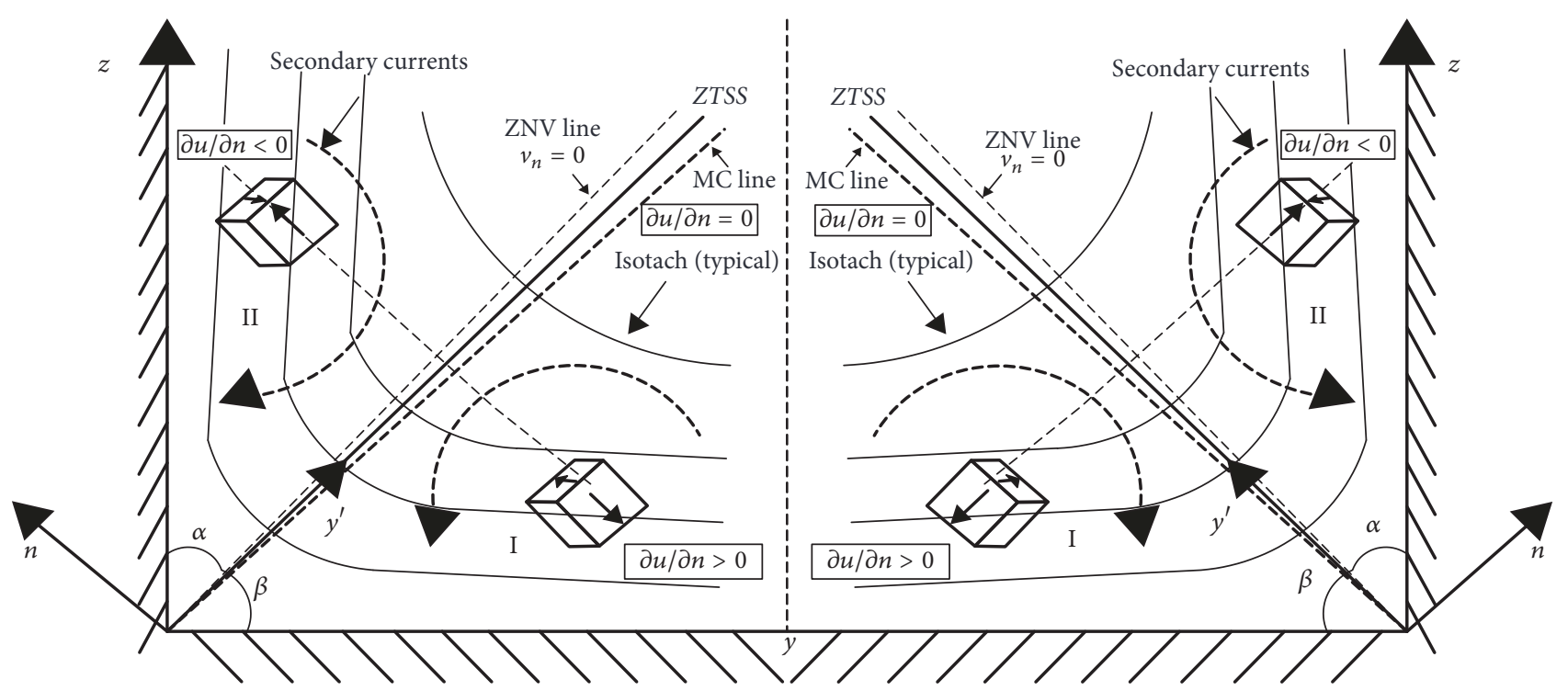

FIGURE 1: Relationships between mean velocity contours, secondary currents, and division lines in a rectangular open channel flow.

where $\mu$ is the dynamic viscosity, $n$ represents the direction normal to the division line interface, and $v_{n}$ is the velocity component of secondary flow normal to the division line. The Reynolds shear stress can be modeled by drawing an analogy with the coefficient of molecular viscosity in Stokes' law in the following form:

$$
\overline{-u^{\prime} v_{n}^{\prime}}=v_{t} \frac{\partial u}{\partial n}
$$

where $v_{t}$ is the turbulent kinematic viscosity. Thus, (4) can be rewritten as

$$
\tau=\left(\mu+v_{t}\right) \frac{\partial u}{\partial n}-\rho u v_{n}
$$

Hence, we can infer based on (6) that meeting the ZTSS condition of $\tau=0$ requires that the following conditions are simultaneously satisfied:

$$
\begin{aligned}
& \text { (1) } \partial u / \partial n=0 . \\
& \text { (2) } v_{n}=0 .
\end{aligned}
$$

The first condition states that either the gradient of $u$ along the division line defined in (6) is approximately equal to zero or that $u$ is at a maximum at the line. The second condition states that no secondary fluid flow penetrates the division line defined in (6) and is therefore parallel to the division line.

It is therefore necessary to identify the locations where $\partial u / \partial n=0$ and $v_{n}=0$ in the flow region. This can be addressed by plotting the isovels in the flow region according to the $y$ and $z$ directions, as shown in Figure 1. Experimental research conducted by Melling and Whitelaw [23] indicates that the isovel lines in open rectangular channel flow bulge markedly towards the corners. Therefore, we can calculate the curvature $k_{c}$ of every point along an isovel and determine the points of maximum curvature (MC). We then plotted a bold dashed line linking the MC points on the isovels in Figure 1, which is denoted as the MC line. In addition, the secondary current vectors were determined, and a fine dashed line indicating the line along which $v_{n}=0$, denoted as the ZNV line, was plotted in Figure 1 accordingly. These lines divide the flow region into regions I and II. We note that $\partial u / \partial n>0$ in flow region I and $\partial u / \partial n<0$ in region II.

The procedure for determining the locations of division lines in an open channel flow by considering the conditions of ZTSS based on the simultaneous satisfaction of conditions 1 and 2 can be summarized as follows:

(i) First, plot an isovel graph $u / U_{m}$, where $U_{m}$ is maximum velocity in flow. Next, fit the isovel lines to polynomials as

$$
y=f(z)
$$

(ii) Calculate $k_{c}$ for every point along an isovel as follows:

$$
k_{c}=\frac{\left|y^{\prime \prime}\right|}{\left(1+y^{\prime 2}\right)^{3 / 2}} \text {. }
$$

(iii) Select the MC points for each isovel, and obtain the MC line linking all MC points on the isovels, which approximately satisfies the condition $\partial u / \partial n=0$.

(iv) The secondary current vectors are plotted on the same graph and are partitioned according to the ZNV line.

(v) The ZTSS division line is plotted between the ZNV and MC lines. Thus, both $\partial u / \partial n$ and $v_{n}$ are approximately equal to zero along the ZTSS line, which provides a good approximation of the physical division line.

2.3. Experimental Verification of Division Lines in an Open Rectangular Channel. The experiment was conducted in 
TABLE 1: Experimental condition.

\begin{tabular}{lccccrr}
\hline Flow Parameter & $\begin{array}{c}\text { Length } \\
L(\mathrm{~m})\end{array}$ & $\begin{array}{c}\text { Width } \\
b(\mathrm{~m})\end{array}$ & $\begin{array}{c}\text { Depth } \\
h(\mathrm{~m})\end{array}$ & $\begin{array}{c}\text { Aspect ratio, } \\
b / h\end{array}$ & $\begin{array}{c}\sqrt{g R S} \\
(\mathrm{~m} / \mathrm{s})\end{array}$ & $\begin{array}{l}\sqrt{g h S} \\
(\mathrm{~m} / \mathrm{s})\end{array}$ \\
\hline UOW data & 11 & 0.3 & 0.11 & 2.73 & 0.025 & 0.033 \\
Nezu et al. [24] data (A1) & 6 & 0.04 & 0.04 & 1 & 0.031 & 0.039 \\
\hline
\end{tabular}

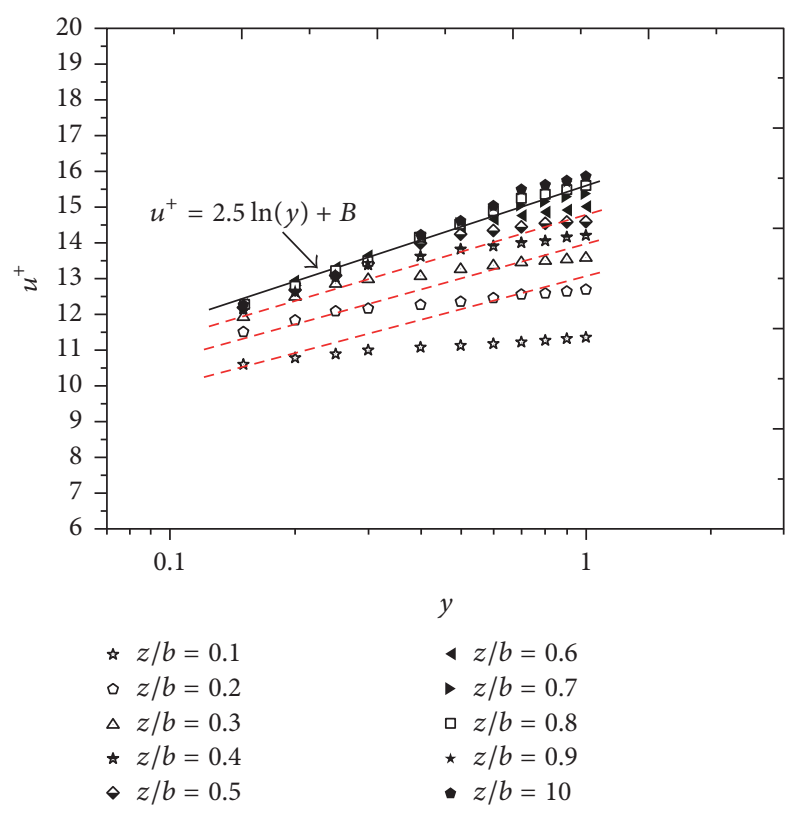

FIGURE 2: Measured velocity distributions along different layers $z$ (where $z=0$ represents the channel bottom) determined relative to the channel depth $b$ plotted in the form of $u^{+}=u /(g R S)^{0.5}$ versus the natural logarithm of the distance from a wall $(y)$ in a smooth rectangular channel (data from Nezu et al. [24]).

a smooth rectangular open channel at the University of Wollongong (UOW). The flume was $10.5 \mathrm{~m}$ long, $h=0.3 \mathrm{~m}$ wide, and $b=0.45 \mathrm{~m}$ deep, and a fully developed uniform flow was established at the test section $6.0 \mathrm{~m}$ downstream from the entrance of channel. A series of velocity profiles were measured using a Dantec two component LDA system. A hot-wire system was used to measure the flow velocity. The detailed experimental conditions are presented in Table 1. The experimental data obtained by Nezu et al. [24] were also examined for comparison.

First, the velocity distributions measured by Nezu et al. [24] and at the UOW along different layers $z$ (where $z=0$ represents the channel bottom) determined relative to the channel depth $b$ were plotted in the form $u^{+}=u / \sqrt{g R S}$ versus the natural logarithm of the distance from a wall (ln $y$ ), as shown in Figures 2 and 3, respectively. As predicted by. (2), the results show that, to a certain extent, the measured velocity in the near wall region follows the solid straight line given in the figures. It is interesting to note that the other dashed lines have an equivalent slope with that of the solid line for data points near the wall, but involve different intercepts. This is particularly evident for the UOW data presented in Figure 3. The identical slope implies that $u_{* 1}$ in (2) is a constant, while the different intercepts imply that $u_{* s}$ in (3) is a variable. Thus, according to the intercepts of each straight line, the results show that $u_{* s}$ increases with increasing $z / b$.

As discussed in Section 2.1, the distance $L_{n}$ along the normal direction from the division line to the boundary can be determined from the calculated values of $u_{* s}$ as $L_{n}=u_{* s}^{2} /(g S)$. Therefore, the upper boundary of the subregion or division line can be obtained, and the results based on the values of $L_{n}$ determined from the intercepts of the straight lines obtained from Figures 2 and 3 are shown in Figures 4(a) and 4(b), respectively, and are plotted relative to the channel width $h$ with respect to $z / b$. Yang and Lim [14] hypothesized that turbulent energy must be transported through the minimum relative distance towards the boundary for dissipation. Thus, the distance $L_{n}$ can also be considered as the energy transfer path. For comparison, the division lines based upon models proposed by different authors are also included in Figure 4. In addition, the ZTSS line is also plotted in Figure 4 . We obtained the ZTSS line according to the procedure presented in Section 2.2 for both the data obtained from Nezu et al. [24] and UOW. According to the outlined procedure, we plotted the isovel graphs $u / U_{m}$, as shown in Figures 5(a) and 5(b) for the data obtained from Nezu et al. [24] and UOW, respectively. All MC points along each isovel were computed according to Eq. (8) using MATLAB, and the MC lines were obtained by linking all the $\mathrm{MC}$ points. The MC line is presented in Figure 5 as a red dashed line (long dashes) running from corner to corner. Meanwhile, the ZNV line, which clearly partitions the two rotating secondary cells, is also plotted in Figure 5 as a red dashed line (short dashes) running from corner to corner. Accordingly, the ZTSS division line lies between the ZNV and MC lines, where both $\partial u / \partial n$ and $v_{n}$ are approximately zero.

As shown in Figure 4, the locations of division lines obtained according to $L_{n}$ and the ZTSS line are quite similar. Such similarity in results obtained from very different methods suggests that division lines exist physically and that the zero-shear stresses can be determined by the velocity contours. A comparison of the various model results indicates that the division line provided by YLM appears to be the most reasonable predictor of division lines relative to the ZTSS and $L_{n}$ results, with a minimum averaged deviation of $18 \%$ and $16 \%$, respectively.

\section{Conclusion}

It is generally assumed that the division of flow regions in a rectangular open channel flow is only a mathematical concept, and no consensus as to whether division lines physically exist has developed. To clarify this, a specially 


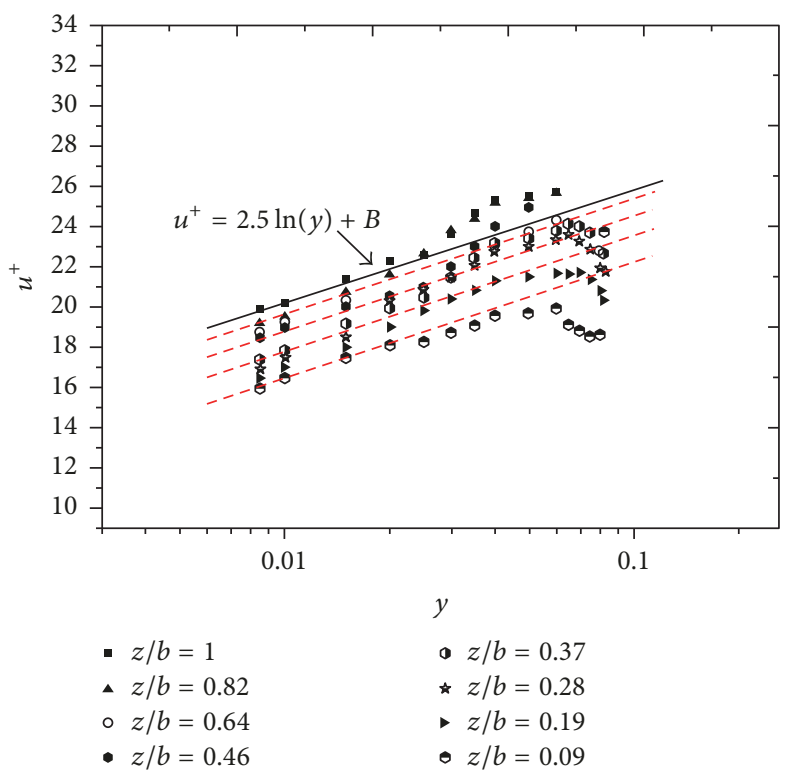

FIGURE 3: Measured velocity distributions along different layers $z$ (where $z=0$ represents the channel bottom) determined relative to the channel depth $b$ plotted in the form of $u^{+}=u /(g R S)^{0.5}$ versus the natural logarithm of the distance from a wall $(y)$ in a smooth rectangular channel (data from UOW).

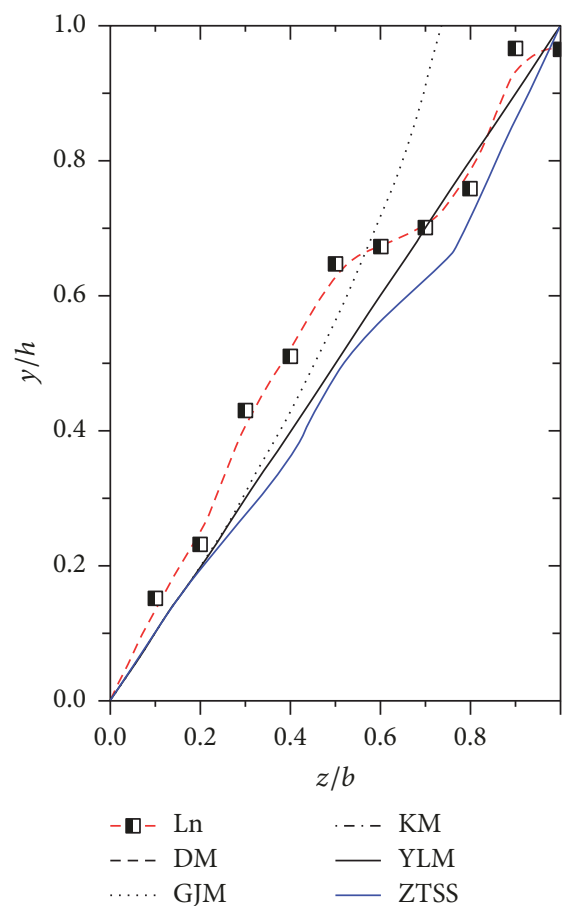

(a)

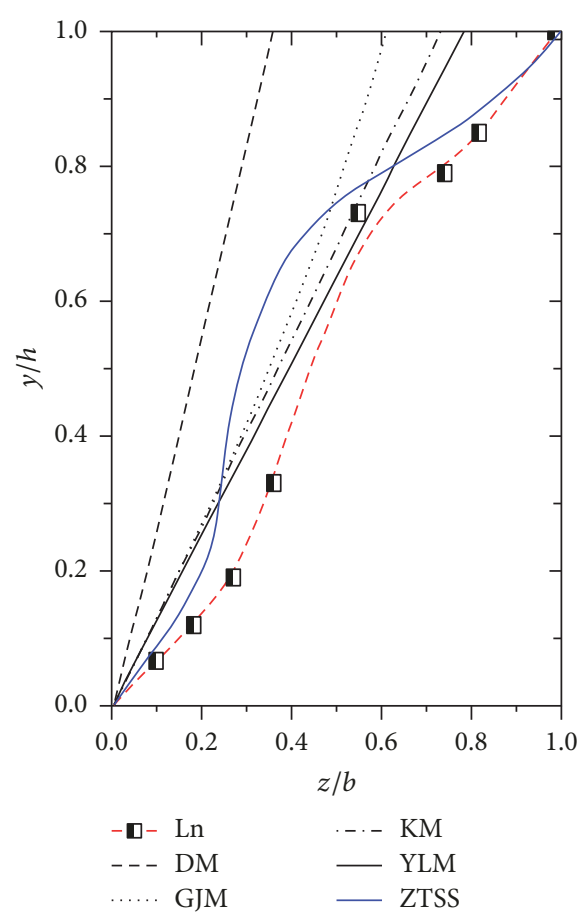

(b)

FIGURE 4: Comparison between measured division lines established according to the ZTSS line and the calculated values of $L_{n}$, and the division lines predicted by DM, GJM, KM, and YLM (data from Nezu et al. [24] (a) and from UOW (b)).

designed apparatus was employed to infer the zero-shear lines from mean velocity distributions in a three-dimensional channel. This investigation made the following contributions, and various conclusions can be drawn.

(1) A clear method was presented to infer the existence of ZTSS lines in a rectangular open channel flow based on velocity contours and secondary current data. This method is a direct and straightforward means of identifying the existence of division lines using the criteria suggested.

(2) The division line can also be determined from the velocity distribution according to the distance $L_{n}$ 


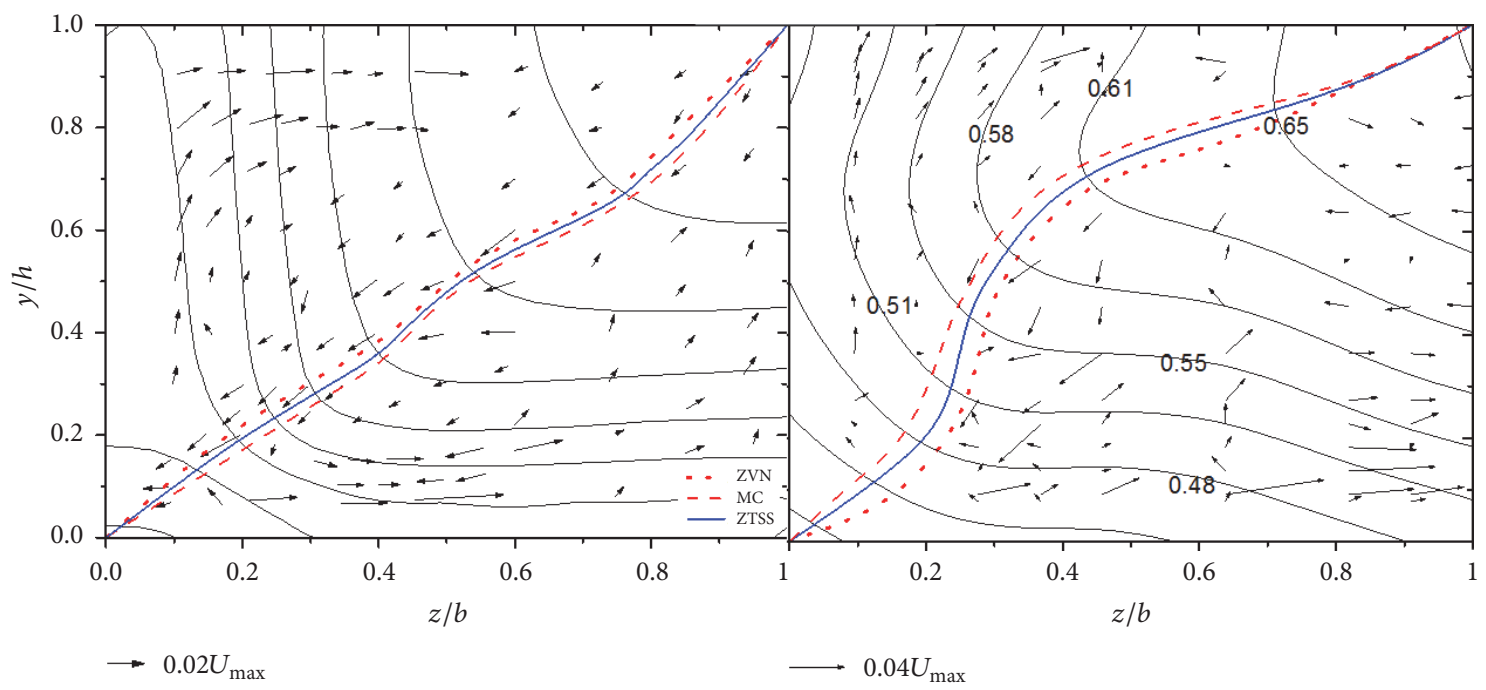

(a)

(b)

FIGURE 5: Measured ZTSS division lines obtained according to the locations of the MC and ZVN lines (data from Nezu et al. [24] (a) and from UOW (b)).

along the normal direction from the division line to the boundary. The experimental division lines obtained from the $L_{n}$ results were very similar to those obtained according to the ZTSS line. Thus, the division line can be determined by identifying the ZTSS and $L_{n}$ locations for a channel flow.

(3) The division lines obtained from the proposed ZTSS and $L_{n}$ methods were compared with those obtained using existing models: KM, DM, YLM, and GJM. The lines obtained by the YLM demonstrated the closest agreement with the division lines obtained from the proposed methods.

\section{Conflicts of Interest}

The authors declare that there are no conflicts of interest regarding the publication of this paper

\section{Acknowledgments}

The authors express gratitude for the financial support from the National Natural Science Foundation of China (Grant no. 51509248) and National Key R\&D Program of China (Grants nos. 2017YFC0403203, 2016YFC0400207, 2017YFD0701000 and 2016YFD200700).

\section{References}

[1] S. Dey, "Sediment pick-up for evolving scour near circular cylinders," Applied Mathematical Modelling, vol. 20, no. 7, pp. 534-539, 1996.

[2] J. Guo, "Modified log-wake-law for smooth rectangular open channel flow," Journal of Hydraulic Research, vol. 52, no. 1, pp. 121-128, 2014.
[3] J. Guo, "Sidewall and non-uniformity corrections for flume experiments," Journal of Hydraulic Research, vol. 53, no. 2, pp. 218-229, 2015.

[4] J. B. Leighly, "Toward a theory of the morphologic significance of turbulence in flow of water in streams," University of California Publications in Geography, vol. 6, no. 1, pp. 1-22, 1932.

[5] C. L. Chiu and J. D. Chiou, "Structure of 3-D flow in rectangular open channels," Journal of Hydraulic Engineering, vol. 112, no. 11, pp. 1050-1068, 1986.

[6] C.-L. Chiu and D. W. Murray, "Variation of velocity distribution along nonuniform open-channel flow," Journal of Hydraulic Engineering, vol. 118, no. 7, pp. 989-1001, 1992.

[7] C.-L. Chiu and C. A. A. Said, "Maximum and mean velocities and entropy in open-channel flow," Journal of Hydraulic Engineering, vol. 121, no. 1, pp. 26-35, 1995.

[8] I. Nezu and H. E. D. Nakagawa, Turbulence in open-channel flows, Balkema, Rotterdam, Netherlands, 1993.

[9] G. H. Keulegan, "Laws of turbulent flow in open channels," Journal of Research of the National Bureau of Standards, vol. 21, no. 6, pp. 707-741, 1938.

[10] H. A. Einstein, Formulas for the transportation of bed load, vol. 107, American Society of Civil Engineers, 1942.

[11] N. Chien and Z. Wan, Mechanics of Sediment Transport, American Society of Civil Engineers, Reston, VA, USA, 1999.

[12] A. Daido, "Effect of aspect ratio of channel and sediment density on transport phenomena," in Proceedings of the Int. Symp. On Hydraulic Research in Nature, pp. 5-7, Chinese Hydraulic Engineering Society, Beijing, China, 1992.

[13] J. Guo and P. Y. Julien, "Shear stress in smooth rectangular openchannel flows," Journal of Hydraulic Engineering, vol. 131, no. 1, pp. 30-37, 2005.

[14] S.-Q. Yang and S.-Y. Lim, "Mechanism of energy transportation and turbulent flow in a 3D channel," Journal of Hydraulic Engineering, vol. 123, no. 8, pp. 684-692, 1997.

[15] S.-Q. Yang, Y. Han, P. Lin, C. Jiang, and R. Walker, "Experimental study on the validity of flow region division," Journal of Hydro-environment Research, vol. 8, no. 4, pp. 421-427, 2014. 
[16] Y. Han, S.-Q. Yang, N. Dharmasiri, and M. Sivakumar, "Effects of sample size and concentration of seeding in LDA measurements on the velocity bias in open channel flow," Flow Measurement and Instrumentation, vol. 38, pp. 92-97, 2014.

[17] Y. Han, S.-Q. Yang, N. Dharmasiri, and M. Sivakumar, "Experimental study of smooth channel flow division based on velocity distribution," Journal of Hydraulic Engineering, vol. 141, no. 4, Article ID 06014025, 2015.

[18] L. Prandtl, "Bericht uber untersuchungen zur aubgebildeten turbulenz," Zeitschrift für Angewandte Mathematik und Mechanik, vol. 5, no. 2, pp. 136-169, 1925.

[19] I. Marusic, J. P. Monty, M. Hultmark, and A. J. Smits, "On the logarithmic region in wall turbulence," Journal of Fluid Mechanics, vol. 716, pp. 1-11, 2013.

[20] J. Nikuradse, "Stromungsgesetze in rauhen rohren," VDI-Forsch, p. 361, 1933.

[21] H. J. Tracy and C. M. Lester, Resistance Coefficients and Velocity Distribution in Smooth Rectangular Channel, USGS Watersupply paper, Washington, DC, USA, 1961.

[22] S.-Q. Yang, S.-Y. Lim, and J. A. McCorquodale, "Investigation of near wall velocity in 3-D smooth channel flows," Journal of Hydraulic Research, vol. 43, no. 2, pp. 149-157, 2005.

[23] A. Melling and J. H. Whitelaw, "Turbulent flow in a rectangular duct," Journal of Fluid Mechanics, vol. 78, no. 2, pp. 289-315, 1976.

[24] I. Nezu, H. Nakagawa, and A. Tominaga, "Secondary currents in a straight channel flow and the relation to its aspect ratio," Turbulent Shear Flows, vol. 4, no. 2, pp. 246-260, 1985. 


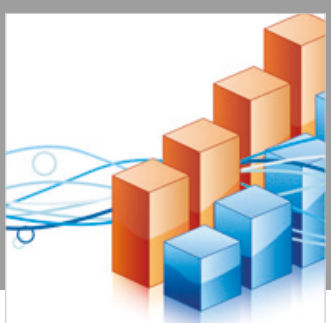

Advances in

Operations Research

\section{-n-m}
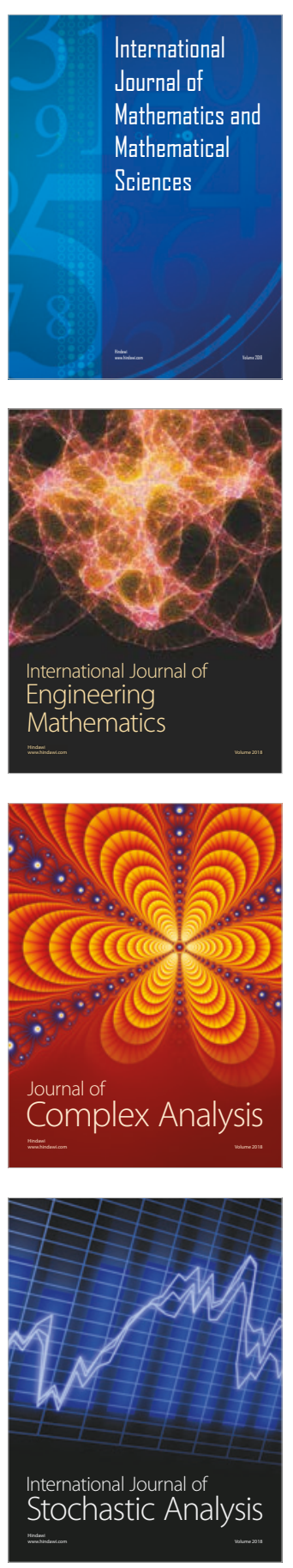
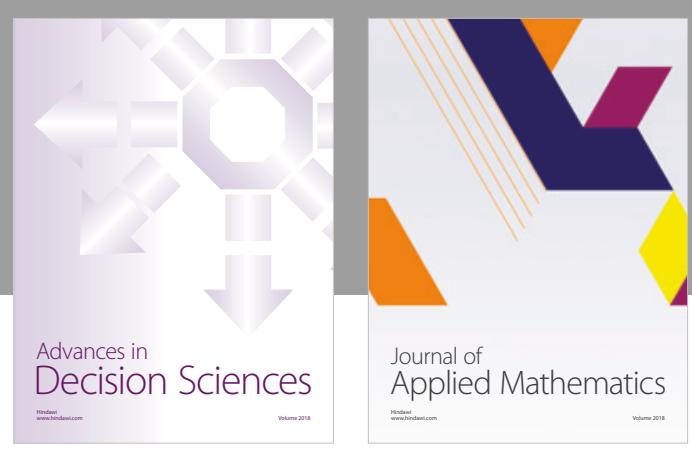

Journal of

Applied Mathematics
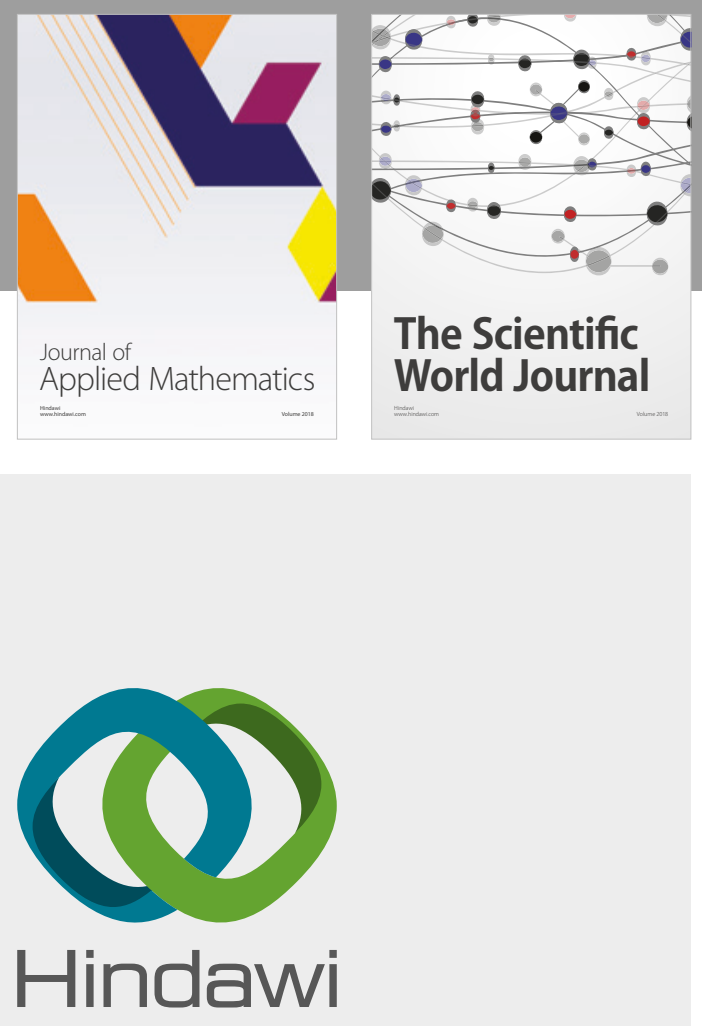

Submit your manuscripts at

www.hindawi.com

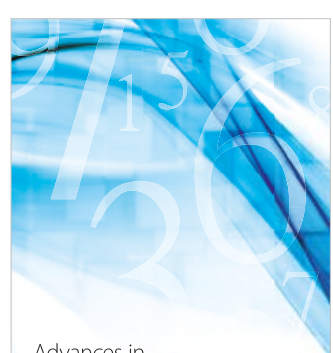

Advances in
Numerical Analysis
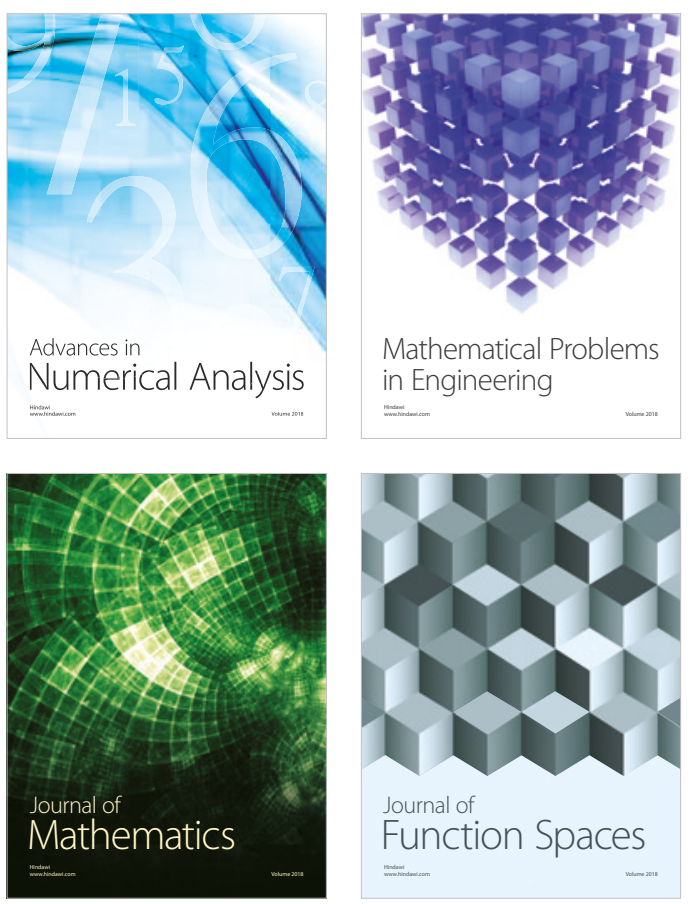

Mathematical Problems in Engineering

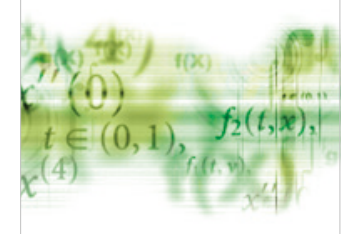

International Journal of

Differential Equations

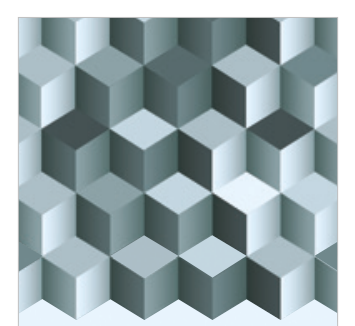

Journal of

Function Spaces

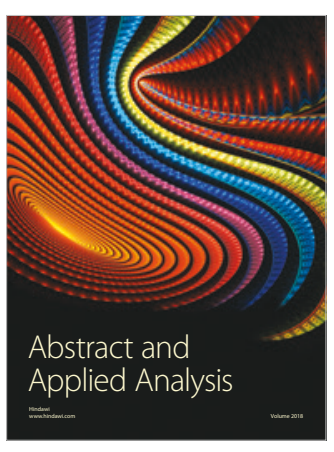

The Scientific

World Journal

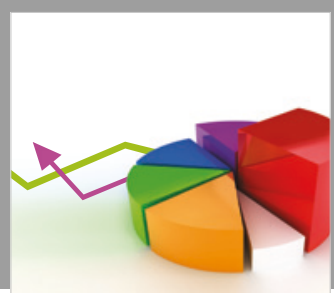

Journal of

Probability and Statistics
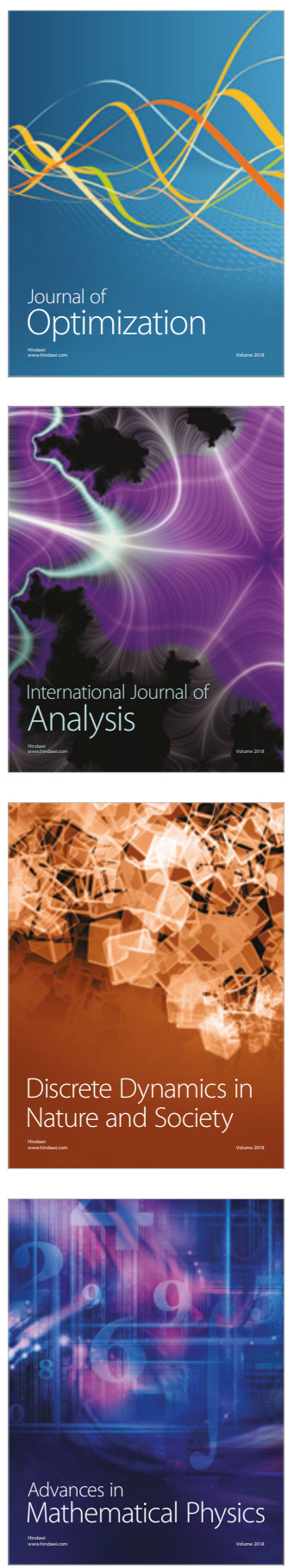